\title{
Lambda point thermodynamics of confined liquid helium: negative surface specific heat
}

\author{
R. A. Ferrell \\ Center for Theoretical Physics of the Department of Physics, University of Maryland \\ College Park, Maryland 20742, USA \\ E-mail: rferrell@muppetn.umd.edu \\ J. K. Bhattacharjee \\ Indian Association for the Cultivation of Science, Jadavpur, Calcutta 700 032, India
}

\begin{abstract}
We present some theoretical considerations that are relevant to the forthcoming measurements in microgravity of the specific heat of confined liquid helium (the flight experiment «CHEX»). Primary attention is devoted to the suppression near the boundaries, the «negative surface specific heat». Above the lambda point, we compute this to lowest order in the $\varepsilon$-expansion: below the lambda point our discussion is more restricted.
\end{abstract}

PACS: 64.60.Ht, 67.40.Hf, 67.40.Pm

The forthcoming measurements in microgravity of the specific heat of liquid ${ }^{4} \mathrm{He}$ confined between parallel plates (the flight experiment «CHEX») present an important challenge to the general theory of the critical properties of a fluid near the critical point of a second order transition. In the case of liquid He, which is described by a complex order parameter, we have noted [1] that the crossover at the lambda point from three-dimensional to two-dimensional behavior is beyond the scope of current theory. It is feasible, however, to develope a theoretical prediction of the temperature dependence in the crossover region by means of an interpolation [1] based on the «negative surface specific heat» outside the crossover region. In this paper we present a computation of this effect above the lambda point to lowest order in the $\varepsilon$-expansion. Because of space limitation, we provide only a quite incomplete discussion of the much more complicated theory below the lambda point.

It is generally accepted that the thermodynamics of liquid ${ }^{4} \mathrm{He}$ are determined by the Ginzburg-Landau free energy functional

$$
F=\frac{1}{2}|\nabla \Psi|^{2}+\frac{a}{2}|\Psi|^{2}+\frac{b}{4}|\Psi|^{4}
$$

provided that the effects of the fluctuations of the complex order parameter, $\Psi$, are properly included. In this short note our attention will be directed mainly to the critical specific heat in the normal state, that is, at temperatures $T$ greater than the lambda temperature $T_{\lambda}$. After a short review of the contributions of the order parameter fluctuations to the bulk specific heat, we will study how these contributions are reduced by the suppression of the fluctuations that results from the boundary condition at the walls of the confining vessel. This fractional reduction, relative to the bulk value, can be regarded as a «negative surface specific heat», and can be expected to be of the form $A \xi S / V$, proportional to the product of the order parameter correlation length times the surface to volume ratio. The primary goal of the first part of this paper is to compute the numerical value of the proportionality coefficient $A$. Our method and approach differ somewhat from those of Schmolke et al. [2] and Huhn and Dohm [3].

In the normal state, each component of the order parameter fluctuates about its mean value zero. To simplify the notation, we let $\Psi$ be a real field that denotes one of these components, and decompose it into the sum of plane waves:

$$
\Psi=\frac{1}{\Omega} \sum_{\mathbf{p}} \Psi_{\mathbf{p}} \exp i \mathbf{p} \times \mathbf{x},
$$

where $\Omega$ is the volume of the hypercube in $D$ dimensional space. Restricting the free energy functional to this component alone, and neglecting the quartic term, we have for the volume integration 


$$
\int F d^{D} x=\frac{1}{2} \sum_{\mathbf{p}}\left(p^{2}+a\right)\left|\Psi_{\mathbf{p}}\right|^{2} .
$$

Applying the equipartition theorem and using units in which the temperature times Boltzmann's constant is one, we obtain for the equilibrium thermal average

$$
\left\langle\left|\Psi_{\mathbf{p}}\right|^{2}\right\rangle=\frac{1}{p^{2}+a} .
$$

The bulk thermodynamic free energy is the negative of the logarithm of the partition function

$$
Z=\sum_{\{\Psi\}} \exp \left(-\int F d^{D} x\right),
$$

summed over all configurations of the fluctuating field $\Psi$. The entropy density is therefore

$$
\frac{\partial \ln Z}{\partial T}=-\frac{a^{\prime}}{2}\left\langle\Psi^{2}\right\rangle
$$

where the prime denotes the temperature derivative. In the following discussion, we will ignore the coefficient $a^{\prime} / 2$ and regard $-\left\langle\Psi^{2}\right\rangle$ as representing the entropy. Substitution from Eq. (2) and replacement of the summation by an integration yield

$$
\left\langle\Psi^{2}\right\rangle=\frac{1}{\Omega} \sum_{\mathbf{p}}\left\langle\left|\Psi_{\mathbf{p}}\right|^{2}\right\rangle=\frac{1}{(2 \pi)^{D}} \int \frac{d^{D} p}{p^{2}+\kappa^{2}} .
$$

Here we have introduced the inverese correlation length

$$
\xi^{-1}=\kappa=\sqrt{a} .
$$

In anticipation of our later treatment of the surface effect, it is useful to separate one of the Cartesian components of $\mathbf{p}$, say $q$, from the remaining $D-1$ components, $\mathbf{k}$. Substituting

$$
p^{2}=q^{2}+k^{2}
$$

into Eq. (7) and carrying out the integration over $q$ yields

$$
\frac{1}{2 \pi} \int_{-\infty}^{\infty} \frac{d q}{q^{2}+k^{2}+\kappa^{2}}=\frac{1}{2}\left(k^{2}+\kappa^{2}\right)^{-1 / 2} .
$$

Thus,

$$
\left\langle\Psi^{2}\right\rangle=\frac{1}{2(2 \pi)^{D-1}} \int \frac{d^{D-1} k}{\left(k^{2}+\kappa^{2}\right)^{1 / 2}} .
$$

Because differentiation with respect to $\kappa^{2}$ is, except for the factor $a^{\prime}$, the same as differentiation with respect to $T$, the bulk specific heat is proportional to

$$
-\frac{\partial\left\langle\Psi^{2}\right\rangle}{\partial \kappa^{2}}=\frac{1}{4(2 \pi)^{D-1}} \int_{0}^{q_{D}} \frac{d^{D-1} k}{\left(k^{2}+\kappa^{2}\right)^{3 / 2}} .
$$

The Wilson-Fisher $\varepsilon$-expansion theory calculates the specific heat as a Taylor's series in powers of $\varepsilon=4-D$. With enough terms in the truncated power series, a good account can be rendered of the thermodynamics in the actual three-dimensional system, i.e., $\varepsilon=1$. It is found, however, that smaller values of $\varepsilon$ can simulate the lambda-point properties of ${ }^{4} \mathrm{He}$, provided that $n$, the number of components of the order parameter, is taken as a continuous variable and permitted to increase above its natural value of 2 as $\varepsilon$ decreases below 1 . Following this procedure and introducing the Debye cutoff $q_{D}$, we obtain a satisfactory representation of the bulk specific heat from the $\varepsilon \rightarrow 0$ $(D \rightarrow 4)$ limit of Eq. (12). (The neglect of the quartic term in Eq. (1) is justified in this limit because the coefficient, $b$, is proportional to $\varepsilon$.) Thus,

$$
-\frac{\partial\left\langle\Psi^{2}\right\rangle}{\partial \kappa^{2}}=\frac{1}{4(2 \pi)^{3}} \int_{0}^{q_{D}} \frac{d^{3} k}{\left(k^{2}+\kappa^{2}\right)^{3 / 2}}=\frac{1}{4(2 \pi)^{3}} 4 \pi \int_{0}^{q_{D}} \frac{k^{2} d k}{\left(k^{2}+\kappa^{2}\right)^{3 / 2}}=\frac{1}{8 \pi^{2}}\left(\ln \frac{q_{D}}{\kappa}+\text { const }\right)=\frac{1}{16 \pi^{2}}\left(\ln \frac{q_{D}^{2}}{\kappa^{2}}+\text { const }\right) .
$$

This compares well with the experimentally measured specific heat. Because of the very small empirical value of the critical exponent, the logarithm provides a good representation of the temperature dependence.

The above brief review of the bulk specific heat will now serve as a basis for our investigation of the 
effect of imposing the Dirichlet boundary condition at parallel planes separated by $L$ and normal to the direction of the momentum coordinate $q$. Thus, the fluctuation modes are no longer «running waves» but rather «standing» sine waves with quantized momenta

$$
q_{n}=n \frac{\pi}{L},
$$

and spacing $\Delta q_{n}=\pi / L$. Instead of the integration over $q$ we now have the sum

$$
\frac{1}{L} \sum_{n=1}^{\infty} \frac{1}{q_{n}^{2}+k^{2}+\kappa^{2}}=\frac{1}{2 L}\left(\sum_{n=1}^{\infty} \frac{1}{q_{n}^{2}+k^{2}+\kappa^{2}}+\sum_{-\infty}^{n=-1} \frac{1}{q_{n}^{2}+k^{2}+\kappa^{2}}\right)=\frac{1}{2 L} \sum_{-\infty}^{\infty} \frac{1}{q_{n}^{2}+k^{2}+\kappa^{2}}-\frac{1}{2 L} \frac{1}{k^{2}+\kappa^{2}}
$$

To evaluate the first term in Eq. (15) it is convenient to introduce

$$
Q=\frac{L}{\pi}\left(k^{2}+\kappa^{2}\right)^{1 / 2} .
$$

In terms of this variable the required sum is the following meromorphic function, which has a second order pole at $Q=0$ and first order poles at $i$ times the non-zero integers:

$$
\begin{gathered}
\frac{1}{2 L} \sum_{n=-\infty}^{\infty} \frac{1}{q_{n}^{2}+\left(\pi^{2} / L^{2}\right) Q^{2}}=\frac{L}{2 \pi^{2}} \sum_{n=-\infty}^{\infty} \frac{1}{n^{2}+Q^{2}}= \\
\quad=\frac{L}{2 \pi Q} \operatorname{coth}(\pi Q)=\frac{\operatorname{coth}(\pi Q)}{2\left(k^{2}+\kappa^{2}\right)^{1 / 2}} .
\end{gathered}
$$

We need this expression for large values of $Q$, for which

$$
\operatorname{coth}(\pi Q) \approx 1-2 \exp (-2 \pi Q) .
$$

The last term expresses the perturbing effect of the overlap of the two penetration regions that extend from the opposite sides into the interior of the fluid. With

$$
\pi Q \geq L k=\frac{L}{\xi}>>1,
$$

we see that Eq. (17) is nothing other than Eq. (10), except for a very small error of the order of, or less than, $\exp (-2 L / \xi)$. This error is evidently such as might be expected from the general «rule of thumb» [4] that any disturbance to the squared order parameter should heal at distances $l>>\xi / 2$ and die out as $\exp (-2 l / \xi)$. According to Eq. (18): keeping $\xi$ below the upper bound

$$
\xi_{u . b .}=\frac{1}{2} L
$$

should hold the error to less that $4 \%$.

With the neglect of the exponentially small error in Eq. (18), the final term in Eq. (15) represents the only remaining effect of the Dirichlet boundary conditions. The surface contribution to the entropy is, therefore,

$$
-\left\langle\Psi^{2}\right\rangle_{\text {Dir }}=\frac{1}{2 L} \frac{1}{(2 \pi)^{D-1}} \int \frac{d^{D-1} k}{k^{2}+\kappa^{2}} .
$$

Setting $D-1$ again equal 3 , we find for the surface specific heat

$$
\begin{gathered}
-\frac{\partial\left\langle\Psi^{2}\right\rangle_{\operatorname{Dir}}}{\partial \kappa^{2}}=-\frac{1}{2 L} \frac{1}{(2 \pi)^{3}} \int \frac{d^{3} k}{\left(k^{2}+\kappa^{2}\right)^{2}}= \\
=-\frac{1}{4 \pi^{2} L} \int_{0}^{\infty} \frac{k^{2} d k}{\left(k^{2}+\kappa^{2}\right)^{2}}=-\frac{1}{4 \pi^{2} L} \frac{\pi}{4 \kappa}=-\frac{1}{16 \pi^{2}} \frac{\pi \xi}{L} .
\end{gathered}
$$

Gathering together Eqs. (13) and (21), we have for the effect of the Dirichlet boundary condition at the walls

$$
\begin{gathered}
-\frac{\partial\left[\left\langle\Psi^{2}\right\rangle+\left\langle\Psi^{2}\right\rangle_{\text {Dir }}\right]}{\partial \kappa^{2}}=\frac{1}{16 \pi^{2}}\left[\ln \left(\frac{q_{D}^{2}}{\kappa^{2}}\right)+\text { const }-\pi \frac{\xi}{L}\right]= \\
=\frac{1}{16 \pi^{2}}\left[\ln \left(\frac{q_{D}^{2}}{\kappa^{2}}\right)+\text { const }-\frac{\pi}{2} \frac{S \xi}{V}\right] .
\end{gathered}
$$

The final form of this equation is a generalization to an arbitrary shape of the fluid sample, and is valid provided that $\xi$ is small in comparison with the characteristic dimensions of the vessel. The coefficient of $S \xi / V$ that is predicted by the above simple theory is evidently

$$
A_{\mathrm{th}}=\frac{\pi}{2} \approx 1.57 \text {. }
$$

This theoretical result can he compared with some recent measurements by Mehta and Casparini [5] for confinement between plane walls of separation equal to $L=2110 \AA$ and $L=5040 \AA$. We limit our attention to the thinner of these fluid layers, for which the surface effect is bigger and thus easier to 
read off from the display of the data in their Fig. 2. To limit the overlap error to less than $4 \%$, Eq. (20) requires $\xi<\xi_{u . b .}=1055 \AA$. With the correlation length given by

$$
\xi(t)=\xi_{0} t^{-v} .
$$

where $\xi_{0}=1.4 \AA$ and $v=0.672$, this implies $t>0,5 \cdot 10^{-4}$. In fact, from the lower curve of Mehta and Casparini Fig. 2 we find that the size of the drop below the bulk specific heat fits very well the temperature dependence $t^{-v}$, with a deviation of $4 \%$ first showing up at $t=0,5 \cdot 10^{-4}$ and increasing rapidly at lower temperatures.

It remains only to deduce the coefficient $A$ from their data, for which one choice of temperature in the surface scaling range will suffice. At $t=10^{-4}$ the negative surface specific heat is evidently $\Delta C=-3.85 \mathrm{~J} /$ mole. $\mathrm{K}$. The bulk specific heat is fitted by

$$
C_{\text {bulk }}=B\left(\ln \frac{q_{D}^{2}}{\kappa^{2}}+\text { const }\right)
$$

with $B=4.01 \mathrm{~J} /$ mole $\cdot \mathrm{K}$. Including the surface effect gives the total as

$$
C_{\text {tot }}=C_{\text {bulk }}+\Delta C=B\left(\ln \frac{q_{D}^{2}}{\kappa^{2}}+\operatorname{const}+\frac{\Delta C}{B}\right),
$$

of the same form as Eq. (23), which permits the identification

$$
A_{\exp } \frac{\xi}{L / 2}=-\frac{\Delta C}{B}=0.960
$$

According to Eq. (25), $\xi=682 \AA$ at $t=10^{-4}$, so

$$
A_{\text {exp }}=1.49 \text {, }
$$

$5 \%$ below the theoretically predicted value in Eq. (24). This agreement is remarkably close, in view of the approximate nature of the calculation. A more complete theoretical treatment would produce $A_{\mathrm{th}}$ as a Taylor's series in powers of $\varepsilon$, of which Eq. (24) would be the truncated version, stopping at the first term.

An alternative experimental study of the negative surface specific heat, for ${ }^{4} \mathrm{He}$ confined in 8 -micron diameter cylindric pores, has been reported by Coleman and Lipa [6]. The lower half of Fig. 1 in [6] exhibits a $\log$ - $\log$ plot of their $T>T_{\lambda}$ data, which is fitted well by the $t^{-v}$ temperature dependence. But lacking a comparison with the bulk specific heat, as well as relevant information on the dimensional units, we are not able to deduce from their data an independent value for $A_{\exp }$.

For the sake of brevity we now limit our review of the computation of the negative surface specific heat for $T<T_{\lambda}$ to its most salient features, leaving the details and remaining features to be presented elsewhere. For $a<0$ the symmetry with respect to the order parameter is broken and it takes on the mean field value $\Psi_{M F}$ fixed by

$$
\Psi_{M F}^{2}=-\frac{a}{b} .
$$

With $\Psi=\Psi_{M F}+\varphi$, the terms in $F$ quadratic in the fluctuation $\varphi$ are

$$
\frac{a}{2} \varphi^{2}+\frac{3 b}{2} \Psi_{M F}^{2} \varphi^{2}=-a \varphi^{2} \equiv \frac{\kappa^{2}-}{2} \varphi^{2},
$$

where the inverse correlation length is given by

$$
\xi_{-}^{-2}=\kappa_{-}^{2}=2|a|
$$

Imposing the Dirichlet boundary condition at the plane $z=0$ gives the mean field order parameter the Ginzburg-Pitaevsky space dependence

$$
\Psi_{0}(z)=\Psi_{M F} \tanh (z / 2 \xi)
$$

The resulting surface loss in entropy is

$$
\begin{gathered}
\int_{0}^{z} d z\left[\Psi_{0}^{2}(z)-\Psi_{M F}^{2}\right]= \\
=\Psi_{M F}^{2} \int_{0}^{\infty} d z \operatorname{sech}^{2} \frac{z}{2 \xi_{-}}=2 \xi_{-} \Psi_{M F}^{2}=\frac{\xi_{-}}{b} 2|a|=\frac{\kappa_{-}}{b},
\end{gathered}
$$

the temperature differentiation of which yields a surface correction to the temperature independent mean field bulk specific heat coming from the differentiation of $\Psi_{M F}^{2}$ itself. For a more subtle effect of the z-dependence of $\Psi_{0}(z)$ we return to Eq. (31) to find that the coefficient of the quadratic term,

$$
\left[a+3 b \Psi_{0}^{2}(z)\right] \frac{\varphi^{2}}{2}=\frac{\kappa_{-}^{2}}{2}\left(1-\frac{3}{2} \operatorname{sech}^{2} \frac{z}{2 \xi}\right) \varphi^{2},
$$

dips down to negative values close to the boundary. This results in a localized fluctuating mode and discrete energy eigenvalue below the continuum, the consequences of which will be examined elsewhere. 
This work has been supported by a Grant for Basic Research NAG 3-1867, from the National Aeronautics and Space Administration.

1. R. A. Ferrell and J. K. Bhattacharjee, in: Proc. of the Joint $X$ th European and VIth Russian Symposium on Physical Sciences in Microgravity, St. Petersburg, Russia, June 1521, 1997, to be publ.

2. R. Schmolke, A. Wacker, V. Dohm, and D. Frank, Physica B165-166, 575 (1990).

3. W. Huhn and V. Dohm, Phys. Rev. Lett. 61, 1368 (1988).
4. R. A. Ferrell, J. Phys. (Paris) 32, 85 (1971).

5. S. Mehta and F. M. Gasparini, in: Proc. of the 21st Intern. Conf. on Low Temperature Physics, Prague, August 8-14, 1996, S. Danis. V. Gregor, and K. Zareta (eds.), Part Sl. Czech. J. Phys., vol. 46, Suppl. Sl, (1996), p. 173

6. M. Coleman and S. A. Lipa, in: Proc. of the 21st Intern. Conf. on Low Temperature Physics, Prague, August 8-14, 1996, S. Danis, V. Gregor, and K. Zareta (eds.), Part Sl. Czech. J. Phys., vol. 46, Suppl. Sl. (1996), p. 183. 